\title{
PRECISE RECEIVER CLOCK OFFSET ESTIMATIONS ACCORDING TO EACH GLOBAL NAVIGATION SATELLITE SYSTEMS (GNSS) TIMESCALES
}

\author{
Thayathip Thongtan \\ National Institute of Metrology, Thailand \\ E-mail: thayathip@nimt.or.th \\ Pawit Tirawanichakul and Chalermchon Satirapod* \\ Department of Survey Engineering, Chulalongkorn University, Thailand \\ E-mails: tirawanichakul.pawit@gmail.com, *chalermchon.s@chula.ac.th (corresponding \\ author)
}

\begin{abstract}
Each GNSS constellation operates its own system times; namely, GPS system time (GPST), GLONASS system time (GLONASST), BeiDou system time (BDT) and Galileo system time (GST). They could be traced back to Coordinated Universal Time (UTC) scale and are aligned to GPST. This paper estimates the receiver clock offsets to three timescales: GPST, GLONASST and BDT. The two measurement scenarios use two identical multi-GNSS geodetic receivers connected to the same geodetic antenna through a splitter. One receiver is driven by its internal oscillators and another receiver is connected to the external frequency oscillators, caesium frequency standard, kept as the Thailand standard time scale at the National Institute of Metrology (Thailand) called UTC(NIMT). The three weeks data are observed at 30 seconds sample rate. The receiver clock offsets with respected to the three system time are estimated and analysed through the geodetic technique of static Precise Point Positioning (PPP) using a data processing software developed by Wuhan University - Positioning And Navigation Data Analyst (PANDA) software. The estimated receiver clock offsets are around 32, 33 and 18 nanoseconds from GPST, GLONASST and BDT respectively. This experiment is initially stated that each timescale is inter-operated with GPST and further measurements on receiver internal delay has to be determined for clock comparisons especially the high accuracy clock at timing laboratories.
\end{abstract}

Keywords: System time, receiver clock offset determination, time comparisons, precise point positioning technique.

\section{INTRODUCTION}

The design principle of navigation satellite system is interoperable though it could be operated independently. The bases of interoperability for multiple GNSS are reference coordinate system and time system. Each navigation satellite coordinate system has its own descriptions which are aligned to the International Terrestrial Reference System (ITRS) determined by the International Earth Rotation and Reference Systems Service (IERS). Each GNSS timescale description is unique in terms of system timescale generation and methods to steer their ensemble clocks in order to align their timescale to the international reference timescale; UTC. Same geodetic and timing references should be referred for all GNSS users 
in order to have consistency in definition. This is beneficial for GNSS reference stations and users in many applications to avoid or compensate systematic errors, Yang (2014) and Huang et al. (2015).

Satellite navigation system is a platform for position determination and time comparison systems. The position is determined from ranges where the time differences between satellites and a user are obtained. The range (so called pseudo-range) between satellites and a user is measured at the received time, $t$. The satellite position is computed at the transmission time, $\mathrm{t}-\tau$. Navigation satellite system timescale is generated from their own timing centre from composite clocks where the timescale at the timing centre is traceable to UTC timescale by satellite common-view links. The time offset between the navigation satellite system time and UTC time is determined; $\Delta \mathrm{t}$, hence, the navigation satellite system time is synchronised with UTC at a certain level of a defined threshold. This technique is called time transfer and is used in the time comparisons where clocks are in remote distances, Teunissen and Kleusberg (1998). The best measurement capability of time and frequency comparisons at NIMT for $5 \mathrm{MHz}$ and $10 \mathrm{MHz}$ output from frequency standards using common-view technique contains its measurement uncertainty at $2.1 \times 10^{-13} \mathrm{~Hz} / \mathrm{Hz}$ determining from the comparison baseline length of less than 1,000 kilometres from NIMT and at the averaging time at 1 day. This determination is by applying the GPS broadcast navigation message and the GPS code observations BIPM KCDB (2017).

Navigation satellite system timescale is specifically analysed to determine performances of their timescale in terms of offset, accuracy and stability, Yang (2014). Their control centre will correct the navigation satellite timescales to UTC scale and later this correction will be uploaded to the satellite vehicles. The International Bureau of Weights and Measures (BIPM) provide recommendations to simultaneously correct for the GPST and GLONASST to be in accordance with UTC, BIPM (2017).

This experiment is conducted by using a GNSS geodetic receivers for time transfer or time comparisons are increasing together with the geodetic processing data of PPP are in demand for several location base services in Thailand. With this PPP approach, it allows larger net work of time link in comparisons to differential positioning technique, improves both positioning and timing accuracy due to more observed satellites, Huang et al. (2015), as well as is able to include GNSS observation data to the national geodetic network for both positioning and time synchronisations, especially making a ground station network at different locations linked to calibrated timescale at the national timing laboratory such as UTC(NIMT) in Thailand.

\section{TIME SYSTEMS}

The basis of civilian time keeping is the atomic timescale namely UTC and GPST. NIMT maintains and disseminates national timescale of UTC(NIMT). It is realised from three caesium beam frequency standards with high performance tube (5071A-001). The time link with the BIPM uses the GPS pseudorange observations by GPS common-view method BIPM (2017), GPS ephemeris is obtained from broadcast navigation messages and reference system of WGS84 is applied for the site coordinate. The UTC(NIMT) is kept with respect to UTC within 100 nanoseconds. The frequency stability, determined from Allan deviation, is < $2.5 \times 10^{-13}$ in 1 day. $<1.1 \times 10^{-13}$ in 5 days and $<5.0 \times 10^{-15}$ in 30 days.

The atomic time is the uniform time scale on the Earth and is kept by atomic clocks. UTC is generated by the BIPM. The clock information kept by each laboratory clocks, UTC $(\mathrm{k})$ and GPST or time broadcasted from each satellite observed at the timing laboratories are 
gathered from around 400 clocks distributed in 70 laboratories worldwide Teunissen and Kleusberg (1998), BIPM (2017).

The International Atomic Time (TAI) scale is established from this clock information together with a set of primary frequency standard kept by around 11 timing institutes. This is to ensure that the time interval unit of TAI second is kept closed to one International System of Units (SI) second. TAI is a continuous time scale and does not relate to the rotation of the Earth because the Earth's is rotating slower. The Earth Orientation Parameters (EOP) is collected by the International Earth Rotation Service (IERS) Teunissen and Kleusberg (1998), BIPM (2017), IERS (2017).

Leap second is added to the TAI time scale and produces the UTC. This makes TAI runs at the same rate with UTC where UTC is synchronised closely; not more than 0.9 seconds, with the time determined by the Earth's rotation Teunissen and Kleusberg (1998).

GPST is an atomic time system; not adjusted to the leap second, which commenced at 00:00 hrs UTC on 6 January 1980. GPS time is restarted each week (Sat/Sun night). Since January 2017, 00:00 hrs UTC, TAI is ahead of UTC by 37 seconds, Equation (1). The leap second was inserted on 31 December 2016 at UTC 23:59:60. TAI is ahead of GPST by 19 seconds, Equation (2). This is a constant number as both TAI and UTC is a continuous time scale. GPST is ahead of UTC by 18 seconds; Equation (1) is subtracted by Equation (2), as shown in Equation (3). The relationship between TAI, UTC and GPST are specified as IERS (2017):

$$
\begin{aligned}
& \text { TAI-UTC }=37 \text { seconds } \\
& \text { TAI-GPST }=19 \text { seconds } \\
& \text { GPST-UTC }=18 \text { seconds }
\end{aligned}
$$

GLONASST is generated on the basis of GLONASS Central Synchroniser time. It is referenced to UTC through UTC(SU) realisation in Moscow. GLONASST is assigned to be 3-hour ahead of UTC(SU) as defined in Equation (4) UNOOSA (2017).

$$
\text { GLONASST }=\mathrm{UTC}(\mathrm{SU})+3 \text { hours }
$$

GST is realised by the Precise Time Facility (PTF) using an ensemble of 2 H-Maser and 4 caesium frequency standards. GST is traceable to UTC and aligned with GPST definition. GST starts at 00:00 hrs UTC on 22 August 1999 and is currently, 00:00 hrs UTC January 2017, ahead of UTC by 18 seconds UNOOSA (2017), as shown in Equation (5).

$$
\text { GST }- \text { UTC }=18 \text { seconds }
$$

BDT is aligned to the time of National Time Service Center (NTSC), UTC(NTSC). BDT started at 00:00 hrs UTC 1 January 2006, based on the average of 10 composite clocks. BDT is fixed by a time and frequency system at master control station UNOOSA (2017). Since the start epoch, BDT is ahead of TAI by 37 seconds; hence, leap second is not taken into account, as shown in Equation (6).

$$
\mathrm{BDT}-\mathrm{TAI}=37 \text { seconds }
$$

\section{RECEIVER CLOCK OFFSET DETERMINATION}

Some commercial GNSS receivers display each system time differences where GPST is assigned as a master clock. The time offset and time drift between each GNSS system time is shown Trimble (2017). This paper shows the time difference between the receiver clock; where timescale is UTC(NIMT) and each GNSS system time, in order to evaluate determine the characteristics of each GNSS system timescale. The receiver clock offsets are compared 
when the receiver internal and external clocks are in operations. The performance determination includes: offsets, accuracy and stability of navigation satellite system time and UTC(NIMT).

The receiver clock offset determination is by observing GNSS signals from a high precision geodetic receiver (Trimble Net-R9) and a geodetic antenna (Trimble Zephyr Geodetic, TRM41249.00 NONE) where the external frequency standard (5071A-001) is inserted to be the receiver reference clock at the frequency of $10 \mathrm{MHz}$ Trimble (2017), Microsemi (2017). This measurement is compared with the current measurement at NIMT. The diagram of this experiment and NIMT measurement set up is as shown in Figure 1.

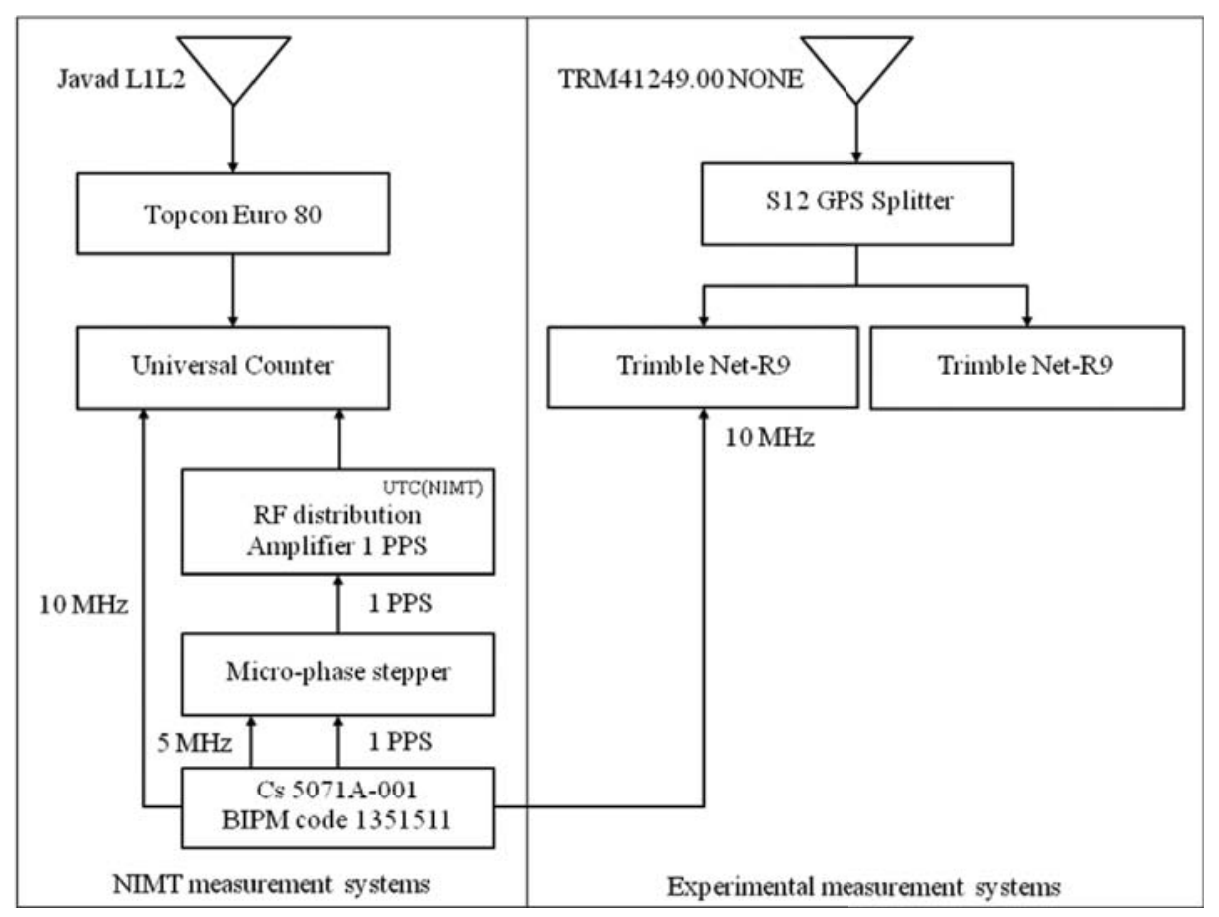

Fig. 1. Measurement diagram of NIMT and experimental setup

GNSS data processing scheme for the daily 30-second observations (RINEX 3.02 files) from this static receiver is PPP. It is a technique to calculate a receiver antenna position where accuracy is to a few centimetres using a single receiver and precise satellite clock and orbit data. In this PANDA processing software, the algorithm applies models of atmosphere parameters, Yang (2014).

The precise satellite and clock orbit data are retrieved from Center for Orbit Determination in Europe (CODE). Two frequency GNSS observables of code and phase could be written as ionosphere-free combination for code and phase observation equation as specified Yang (2014), Teunissen and Kleusberg (1998).

$$
\begin{aligned}
& \mathrm{P}^{\mathrm{j}}(\mathrm{i})=\rho^{\mathrm{j}}(\mathrm{i})+\mathrm{c}\left(\delta \mathrm{t}(\mathrm{i})-\delta \mathrm{T}^{\mathrm{j}}(\mathrm{i})\right)+\mathrm{ZTD}(\mathrm{i}) \cdot \mathrm{M}\left(\mathrm{e}^{\mathrm{j}}(\mathrm{i})\right)+\varepsilon_{\mathrm{p}} \\
& \varphi^{\mathrm{j}}(\mathrm{i}) \cdot \lambda=\rho^{\mathrm{j}}(\mathrm{i})+\mathrm{c}\left(\delta \mathrm{t}(\mathrm{i})-\delta \mathrm{T}^{\mathrm{j}}(\mathrm{i})\right)+\mathrm{ZTD}(\mathrm{i}) \cdot \mathrm{M}\left(\mathrm{e}^{\mathrm{j}}(\mathrm{i})\right)+\mathrm{N}^{\mathrm{j}}(\mathrm{i}) \cdot \lambda+\varepsilon_{\varphi}
\end{aligned}
$$

where

$\mathrm{i}$ and $\mathrm{j}$ are epoch and satellite number;

$\mathrm{P}^{\mathrm{j}}(\mathrm{i})$ and $\varphi^{\mathrm{j}}(\mathrm{i})$-are code and carrier phase observations;

$\rho^{\mathrm{j}}(\mathrm{i})$ is the geometric range between satellite and receiver;

$\delta \mathrm{t}(\mathrm{i})$ and $\delta \mathrm{T}^{\mathrm{j}}(\mathrm{i})$ are receiver and satellite clock offsets; 
$\lambda$ is the ionospheric-free wave length;

$\mathrm{ZTD}(\mathrm{i})$ is the zenith total delay;

$\mathrm{M}\left(\mathrm{e}^{\mathrm{j}}(\mathrm{i})\right)$ is the ZTD mapping function;

$\mathrm{e}^{\mathrm{j}}(\mathrm{i})$ is satellite elevation angle;

$\mathrm{N}^{\mathrm{j}}(\mathrm{i})$ is the ionosphere-free ambiguity;

$\varepsilon_{\mathrm{p}}$ and $\varepsilon_{\varphi}$ are multipath error and observation noise of the code and carrier phase observations.

The parameters to be determined in this experiment are receiver clock offsets and zenith total delay. The receive clock offsets are estimated with static PPP algorithm in respected with GPST, GLONASST and BDT. The results are compared against the globally computed time difference between UTC and GPST, and, UTC and GLONASST available from the BIPM website. Later the timescale performances are evaluated.

This BIPM estimated results of relative timescale of UTC with respected to GPST and GLONASST; and UTC(NIMT) with respected to UTC are determined from NIMT measurement system with a timing receiver, as shown in Figure 1. It is pointed out that the receiver, used data and estimated procedure are not the same with this experimental set up. The current NIMT measurement system used timing receiver where positioning accuracy is less than 1 meter. The observed data are pseudorange from GPS satellites. Processing data is based on relative positioning where visible satellites are taking into the calculation. Satellite position and clock offset are from the satellite broadcast message.

\section{RESULTS}

The receiver clock offsets between receiver clock and each navigation satellite timescales; namely, GPST, GLONASST and BDT, are displayed. They are daily estimation results. These results are from two receivers based on its receiver internal oscillator timescale and external reference timescale of UTC(NIMT). Both estimated results are compared with BIPM estimates for the timescale differences between UTC and UTC(NIMT); GPST; and GLONASST. When the receiver clock offsets are determined, its quality is measured in terms of accuracy and stability in accordance with navigation satellite timescales.

\subsection{Receiver clock offsets}

The daily estimated receiver clock offsets of UTC(NIMT) and each navigation satellite system time are as displayed in Figure 2.

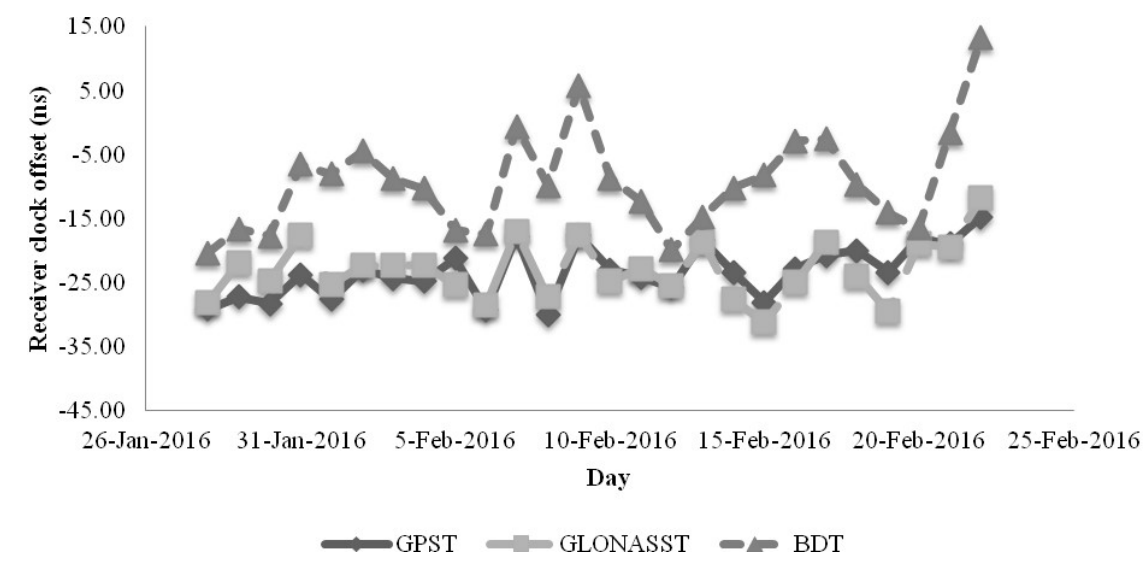

Fig. 2. Receiver clock offset between UTC(NIMT) and each navigation satellite timescales 
Table 1 shows the relative clock offset between UTC(NIMT) and each navigation satellite timescales. The clock offset of UTC(NIMT) and GPST is around $31.7 \mathrm{~ns}$ behind GPST, though its deviation (maximum-minimum of estimated values) is the best amongst other timescales according to the estimates from the 27 days observation data. Relative clock offset of UTC(NIMT) and BDT is averagely good quality in terms of mean but variation is about the same quality with receiver clock offset between UTC(NIMT) and GLONASST.

Table 1. Timescale differences between UTC(NIMT) and navigation satellite timescales

\begin{tabular}{|c|c|c|c|}
\hline & UTC(NIMT)-GPST (ns) & UTC(NIMT)-GLONASST (ns) & UTC(NIMT)-BDT (ns) \\
\hline Mean & -31.7 & -33.1 & 17.6 \\
\hline RMS & 23.6 & 23.4 & 12.0 \\
\hline Min & -35.1 & -40.5 & -22.4 \\
\hline Max & -27.8 & -27.2 & -8.3 \\
\hline
\end{tabular}

The comparisons between the estimated clock offsets from this experimental set up and PPP processing algorithm; and, the estimated clock offsets from BIPM using NIMT current time transfer system is as shown in Figure 3.

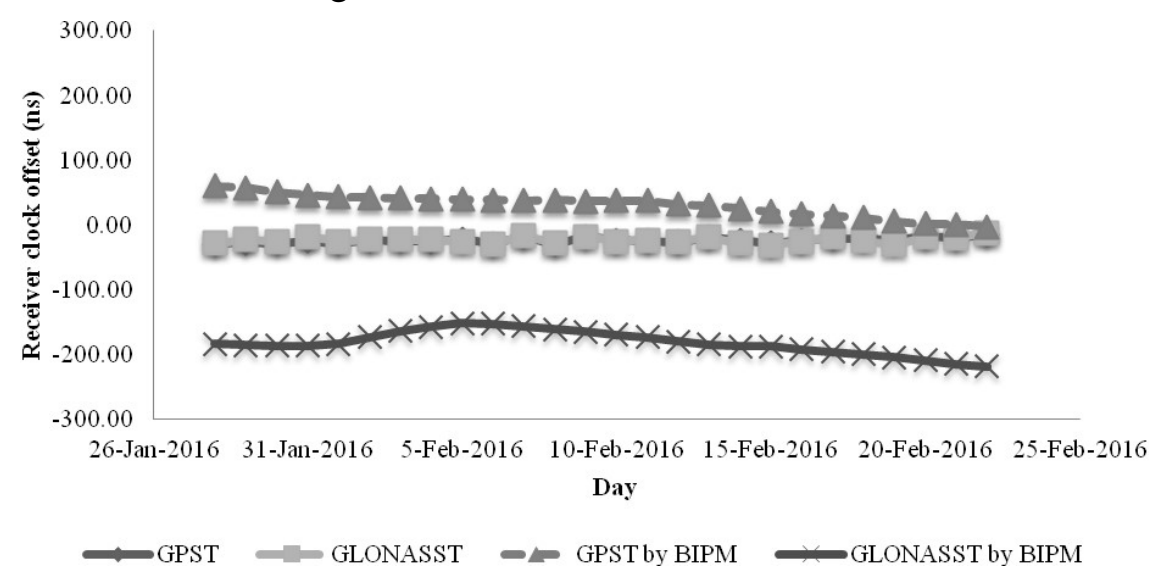

Fig. 3. Comparisons of daily estimated receiver clock offset between UTC(NIMT) with GPST and GLONASST from this experiments to the estimated results from the BIPM

Table 2 shows the BIPM estimated results of UTC(NIMT) with respected to GPST and GLONASST. This estimated receiver clock offsets are in-lined with BIPM estimated results for UTC(NIMT) offset with GPST. The timing offset of the estimated UTC(NIMT) and GLONASST are in contrary.

Table 2. Timescale differences between UTC(NIMT) and navigation satellite timescales by BIPM

\begin{tabular}{|c|c|c|c|}
\hline & UTC(NIMT)-GPST (ns) & UTC(NIMT)-GLONASST(ns) & UTC(NIMT)-BDT (ns) \\
\hline Mean & -31.2 & -181.0 & N/A \\
\hline RMS & 35.6 & 181.9 & N/A \\
\hline Min & -2.5 & -217.9 & N/A \\
\hline Max & -60.7 & -151.4 & N/A \\
\hline
\end{tabular}


The receiver internal clock offsets and each navigation satellite system time are as displayed in Figure 4.

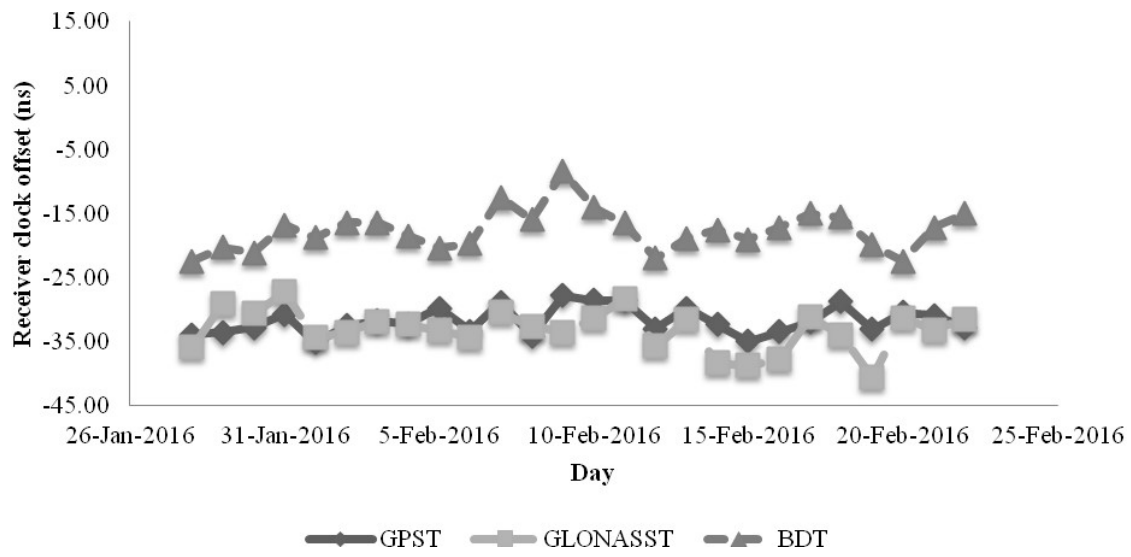

Fig. 4. Timescale differences between receiver internal clock and navigation satellite timescales

Table 3 shows the relative clock offset between receiver internal clock and each navigation satellite timescales. The clock offset of the estimated receiver internal clock and GPST is around $9.2 \mathrm{~ns}$ behind BDT. The variation of the estimated receiver internal clock offsets and GPST is the smallest and is in-lined with estimated receiver internal clock offsets and GLONASST.

Table 3. Timescale differences between receiver internal clock and navigation satellite timescales

\begin{tabular}{|c|c|c|c|}
\hline & $\delta$ t-GPST (ns) & $\delta$ t-GLONASST (ns) & $\delta$ t-BDT (ns) \\
\hline Mean & -23.2 & -22.9 & -9.2 \\
\hline RMS & 31.8 & 33.3 & 17.9 \\
\hline Min & -29.9 & -31.1 & -20.3 \\
\hline Max & -14.7 & -11.7 & 13.2 \\
\hline
\end{tabular}

\subsection{Accuracy}

Receiver clock offsets are determined from each satellite navigation satellite timescales. These values are used to determine its accuracy, where the time offset is the differences between the measured pulse signals and an ideal pulse signals that coincides with UTC, NIST (2017) and Lombardi and Brown (2001) as specified in Equation (9).

$$
f(\text { offset })=\frac{\Delta t}{T}
$$

where

$f$ (offset) is the frequency offset (second/second),

$\Delta t$ is the difference between time interval measurements (second),

$T$ is the measurement period (second).

Table 4 shows accuracy of each navigation satellite timescale relative to receiver clock offsets where UTC(NIMT) or internal clock is applied. The accuracy of the estimated steered 
timescale of UTC(NIMT) with respected to BDT accuracy is $-3.12 \times 10^{-11}$ under $95 \%$ probability and is the highest accuracy amongst three navigation satellite timescales. The accuracy of estimated results from the receiver internal clocks are slightly worse compared to the estimated results from the external clocks. The accuracy of the BDT is the highest in these estimations.

Table 4. Accuracy of relative receiver clock offset to navigation satellite timescales

\begin{tabular}{|c|c|}
\hline & Accuracy $(\mathrm{s} / \mathrm{s})$ \\
\hline UTC(NIMT)-GPST & $-2.65 \times 10^{-10}$ \\
\hline UTC(NIMT) - GLONASST & $-6.33 \times 10^{-10}$ \\
\hline UTC(NIMT) - BDT & $-3.12 \times 10^{-11}$ \\
\hline$\delta \mathrm{t}-\mathrm{GPST}$ & $-6.67 \times 10^{-10}$ \\
\hline dt-GLONASST & $-1.82 \times 10^{-10}$ \\
\hline
\end{tabular}

\subsection{Stability}

The frequency stability is defined using Allan deviation, Riley (2008), specified as Equation

$$
\sigma(\tau)=\sqrt{\frac{1}{2(N-2) \tau^{2}} \sum_{i=1}^{N-2}\left[x_{i+2}-2 x_{i+1}+x_{i}\right]^{2}}
$$

where

$\sigma(\tau)$ is the Allan deviation (second/second),

$x_{t}$ is a series of phase measurements (second),

$N$ is the number of values in the measurement series,

$\tau$ is the equally measurement space (second).

The performance of the estimated values for external frequency standard is not significantly different; though the frequency is slightly stable with respected to GLONASST, from the estimated values for internal frequency standard where GPST, GLONASST and BDT are used as reference timescales. The sample data is within 1 day where the averaging time $(\tau)$ are $16,32,48,64,80,96,112$ and 128 . The results of the relative frequency stability of navigation satellite timescale to the internal and external frequency standard are, as shown in Figure 5 and Figure 6 respectively. 


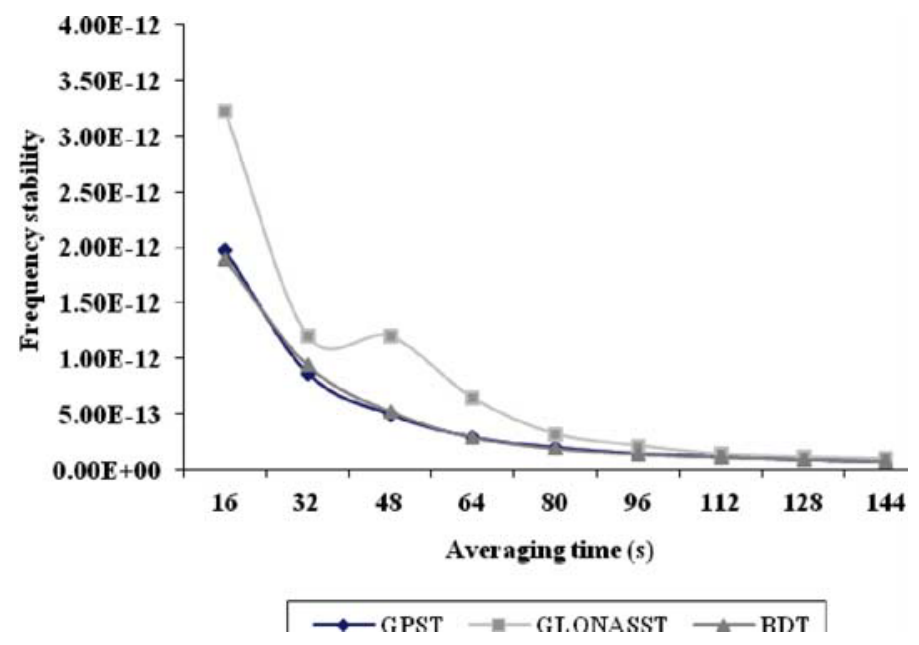

Fig. 5. Frequency stability of receiver internal clock offset to navigation satellite system times

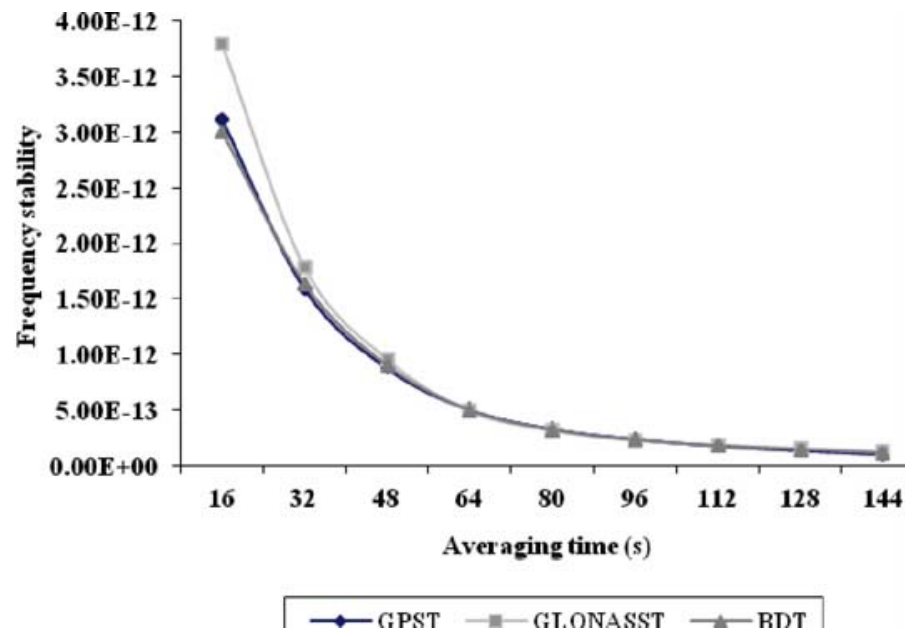

Fig. 6. Frequency stability of receiver external clock offset to navigation satellite system times

\section{CONCLUSIONS}

Navigation satellite timescales are used for international time and frequency comparisons. Geodetic equipment and data processing techniques are used to determine the receiver clock characteristics. With multi-GNSS constellations and multi-system timescales, the time differences between each timescales have to be determined in order to reduce the systematic errors. This experiment determines each timescales differences and its performances including, clock offsets, accuracy and frequency stability. The two receivers; where one relies on internal and another is based on the external clocks, are used to distinguish the differences of the characteristics at the receiver end. The result does not show significant characteristics in accordance with the estimated receiver clock offset from the BIPM. This test results show that other parameters such as receiver internal delay have to be determined and validation techniques are need for receiver clock offsets determination in multi-GNSS constellations. 
Acknowledgements. We thank NIMT management team for allowing us to work on this research. We also thank the department of lands for loaning us their geodetic receivers for three weeks to work on this experiment. All errors are our own.

\section{REFERENCES}

BIPM. (2017) Circular T 349, Retrieved 16 May 2017, from: http://www.bipm.org/jsp/en/TimeFtp.jsp?TypePub=publication.

BIPM KCDB (2017) Calibration and Measurement Capabilities: Time and Frequency; Retrieved 19 August 2017, from: http://kcdb.bipm.org/appendixC/TF/TH/TF TH.pdf

Huang G, Zhang Q, FU W and Guo H. (2015) GPS/GLONASS time offset monitoring based on combined Precise Point Positioning (PPP) approach. Journal of Advances in Space Research, 55, 2950-2960.

IERS. (2017) IERS Conventions, Retrieved 16 May 2017, from http://tai.bipm.org/iers/convupdt/convupdt.html.

Lombardi MA and Brown KH. (2001) NIST Frequency Measurement and Analysis System: Operator's Manual, Colorado, USA.

Microsemi. (2017) Cesium primary frequency standard, Retrieved 20 May 2017, from https://www.microsemi.com/products/timing-synchronization-systems/time-frequencyreferences/cesium-frequency-standards/5071a.

NIST. (2017) NIST Time and Frequency from A to Z, Frequency offset, Retrived 20 May 2017, from https://www.nist.gov/time-and-frequency-services/f.

Riley WJ. (2008) NIST special Publication 1065 Handbook of Frequency Stability Analysis, US government printing office, Washington.

Teunissen PJG and Kleusberg A (Eds.). (1998) GPS for geodesy, Springer-Verlag Berlin Heidelberg, 2nd edition.

Trimble. (2017) Trimble NetR9 GNSS Reference Receiver, Retrieved 20 May 2017, from http://www.trimble.com/Infrastructure/Trimble-NetR9.aspx.

Yang Y. (2014) Training book part 1, International training workshop on BeiDou technology and its applications, Wuhan Hubei, China.

UNOOSA. (2017) Regional reference systems, Retrieved 16 May 2017, from http://www.unoosa.org/oosa/en/ourwork/icg/resources/Regl-ref.html.

Received: 2017-06-01,

Reviewed: 2017-07-25, and 2017-10-09,

Accepted: 2017-12-01. 